\title{
ARTICLE
}

Received 1 Apr 2014 | Accepted 10 Jul 2014 | Published 22 Aug 2014 | Updated 16 Oct 2014

\section{X-ray holography with a customizable reference}

\author{
Andrew V. Martin ${ }^{1, \star}$, Adrian J. D'Alfonso ${ }^{2, \star}$, Fenglin Wang ${ }^{3}$, Richard Bean ${ }^{3}$, Flavio Capotondi ${ }^{4}$, Richard A. Kirian ${ }^{3}$, \\ Emanuele Pedersoli ${ }^{4}$, Lorenzo Raimondi ${ }^{4}$, Francesco Stellato ${ }^{3}$, Chun Hong Yoon ${ }^{3,5}$ \& Henry N. Chapman ${ }^{3,6}$
}

In X-ray Fourier-transform holography, images are formed by exploiting the interference pattern between the $X$-rays scattered from the sample and a known reference wave. To date, this technique has only been possible with a limited set of special reference waves. We demonstrate X-ray Fourier-transform holography with an almost unrestricted choice for the reference wave, permitting experimental geometries to be designed according to the needs of each experiment and opening up new avenues to optimize signal-to-noise and resolution. The optimization of holographic references can aid the development of holographic techniques to meet the demands of resolution and fidelity required for single-shot imaging applications with X-ray lasers.

\footnotetext{
${ }^{1}$ ARC Centre of Excellence for Coherent X-ray Science, School of Physics, The University of Melbourne, Melbourne, Victoria 3010, Australia. ${ }^{2}$ School of Physics, University of Melbourne, Melbourne, Victoria 3010, Australia. ${ }^{3}$ Center for Free-Electron Laser Science, DESY, Hamburg 22607, Germany. ${ }^{4}$ Fermi, Elettra Sincrotrone Trieste, SS 14-km 163.5, Basovizza, Trieste 34149, Italy. ${ }^{5}$ European XFEL GmbH, Albert Einstein Ring 19, Hamburg 22761, Germany.

${ }^{6}$ University of Hamburg, Luruper Chaussee 149, Hamburg 22761, Germany. ${ }^{*}$ These authors contributed equally to this work. Correspondence and requests for materials should be addressed to A.V.M. (email: andrew.martin@unimelb.edu.au).
} 
X -ray Fourier-transform holography $(\mathrm{FTH})^{1-3}$ is a robust coherent imaging technique that has found wide-ranging applications to biology ${ }^{2}$ and material science ${ }^{3}$. The technique is particularly suited to imaging magnetic domains and other magnetic samples because holograms generated with circularly polarized X-rays can be used to separate charge scattering from magnetic scattering ${ }^{3-5}$. X-ray holography has developed into a powerful single measurement technique ${ }^{1,6}$ and has promising applications using X-ray lasers to study structures of radiation-sensitive samples and the structural changes of transient phenomena ${ }^{6-8}$. The definitive feature of holography is the use of a reference wave to facilitate a simple, direct and robust image-reconstruction procedure. However, to date the choice of reference wave in FTH has been restricted to those few cases for which the analytic image-reconstruction procedure is known. This in turn places restrictions on the sample-reference geometry and experimental design. Here we introduce a general imagereconstruction procedure for $\mathrm{FTH}$ data that places minimal restrictions on the reference, enabling a much greater flexibility for the design of $\mathrm{X}$-ray holographic experiments.

The simplest choice of FTH reference is a pinhole in the sample plane, and it was this reference that was used in the first demonstrations of X-ray holography, ${ }^{3,9}$. It is a convenient reference because the hologram is obtained by a single Fourier transform of the measured data. The resolution of the hologram is determined by the diameter of the pinhole, which has the unfortunate consequence of coupling flux and resolution. To improve resolution while maintaining flux, multiple pinholes can be used $^{10}$. However, adjacent pinholes must be sufficiently separated to produce non-overlapping holograms and consequently the field-of-view is used inefficiently. Alternatively, flux and resolution can be decoupled by using extended references ${ }^{1,11-13}$. The image-reconstruction procedure for extended references involves the application of linear operators, such as convolution ${ }^{1}$, linear filters ${ }^{11,12}$ or propagation $^{13}$. However, there are only a handful of special cases for which these reconstruction procedures are known. Notwithstanding the specificity of each approach, the aforementioned methods still leave something to be desired as Fresnel-zone plate references have missing information at low frequencies ${ }^{13}$ and sharp corner references improve resolution only along a few specific directions ${ }^{14}$. A further limitation is that missing data have a significant impact on the results of existing FTH methods. High-intensity X-rays in the forward direction are usually blocked by a beam stop or allowed to pass by gaps in modular detectors, creating regions of missing data that are not accounted for in many existing reconstruction procedures.

There are in fact many more possible reference waves for holographic experiments than have been demonstrated in X-ray holography to date ${ }^{15}$. It is possible to recover images from a large class of reference waves by formulating the holographic image reconstruction within a general framework that leads to a system of linear equations. The linear equations are efficiently and robustly solved by the iterative linear reconstruction using Fourier transforms method (ILRUFT) ${ }^{16}$, which is based on the least-squares conjugate-gradient method ${ }^{17}$. This approach has been successfully applied in the context of electron coherent diffractive imaging (CDI) ${ }^{18}$ and electron ptychography ${ }^{19}$.

Here we develop ILRUFT into a generalized X-ray holography technique and provide a proof-of-principle demonstration that highlights its potential for single-shot femtosecond imaging with $\mathrm{X}$-ray lasers using arbitrary illumination. Our implementation of ILRUFT for X-ray holography allows for the handling of missing data, a feature that is particularly useful for single-shot applications with $\mathrm{X}$-ray lasers, where missing data regions are typically large.

\section{Results}

ILRUFT for X-ray holography. Although the ILRUFT method has been described in detail elsewhere ${ }^{16}$, it will be valuable here to briefly summarize the central ideas before discussing the specific developments required for X-ray holography. The X-ray wave at the exit surface plane of the object $\psi_{\text {exit }}$ can be decomposed into the sum of scattered waves from the object and the reference, as follows

$$
\psi_{\text {exit }}=\psi_{\text {ref }}+\psi_{\text {obj }} .
$$

If the diffraction pattern is composed of multiple exposures then $\psi_{\text {ref }}$ represents the average of all the reference waves, as discussed further in Methods. By taking an inverse Fourier transform of the measured diffraction data (assuming there are no missing data), the autocorrelation of the exit wave $A_{\text {data }}$ is obtained, and may be expressed as

$$
A_{\text {data }}=A_{\text {ref }}+A_{\text {obj }}+C_{\text {ref,obj }},
$$

where $A_{\text {ref }}$ and $A_{\text {obj }}$ are the autocorrelation functions of the reference and object waves, respectively, and $C_{\text {ref,obj }}$ are the crosscorrelations between the reference and the object waves. As explained in detail in ref. 15, when the object is finite, there are large regions of the autocorrelation function where $A_{\mathrm{obj}}=0$, and in this region equation (2) can be reformulated as

$$
A_{\text {data }}-A_{\text {ref }}=C_{\text {ref }, \text { obj }}=\mathcal{M} \psi_{\text {obj }} .
$$

The matrix $\mathcal{M}$ consists of values of $\psi_{\text {ref }}$. Since the reference wave is assumed to be known a priori and the cross-correlations can be reliably extracted from the measured data, both $A_{\text {ref }}$ and $\mathcal{M}$ are known and we can solve equation (3) as a system of linear equations for the unknown scattered wave, $\psi_{\mathrm{obj}}$. Furthermore, an efficient solution to the set of equations can be obtained using the conjugate-gradient least-squares algorithm within the ILRUFT framework, where the action of the matrix $\mathcal{M}$ and its transpose on a vector can be formulated efficiently with Fourier transforms, and $\mathcal{M}$ need never be constructed explicitly.

When the diffraction pattern contains regions of missing data, say, for example, through the use of a beam stop, it is necessary to account for the way in which missing data affect the autocorrelation functions and consequently the set of linear equations need to be modified. We define a binary mask $\mathcal{B}$ that takes the value one on pixels where data were measured and zero otherwise. The linverse Fourier transform of the mask is defined by $P=\mathcal{F}^{-1}[\mathcal{B}]$ (where $\mathcal{F}$ is the Fourier-transform operator). When there are missing data, ILRUFT is applied by making the following substitutions

$$
A_{\text {ref }} \rightarrow P \otimes A_{\text {ref }},
$$

and

$$
\psi_{\text {ref }} \rightarrow P \otimes \psi_{\text {ref }},
$$

where $\otimes$ represents a convolution. As the function $P$ has an extended width, the nonlinear term $P \otimes A_{\text {obj }}$ is no longer confined to the central region of the exit-wave autocorrelation function but expands to contaminate regions where the linear equations would have otherwise been in isolation. We treat this contamination as an additional source of error. If the missing data region is not too extensive, a significant fraction of the linear equation region is not greatly affected by the spreading of the nonlinear information. This and other sources of error (for example, measurement noise) are accommodated through regularization via iteration number in the ILRUFT algorithm. As with CDI techniques, if the extent of the missing data exceeds the width of a speckle (a diffraction fringe with a minimum width), then the reconstructed image will not contain low-frequency information that can affect contrast $^{20}$. The amount of unmeasured object information can 
be characterized by a modal analysis ${ }^{21}$. These considerations and their impact on our generalized holographic approach and the accompanying experimental results will be discussed further below.

The use of a priori knowledge about the scattered wave is a common practice in conventional X-ray CDI, and may also be included in the generalized scheme presented here. Reality and positivity are two object constraints that are commonly used. In the present case we can restrict $\psi_{\text {obj }}$ to be a real function by setting its imaginary component to zero in equation (3). Positivity cannot be incorporated directly into the conjugate-gradient least square iterations because it impedes convergence and alternative non-negative least square solvers that have been reported in the literature 22,23 do not lend themselves to an efficient FFT-based implementation such as ILRUFT. Nevertheless, the conjugategradient iterations can be periodically terminated to enforce positivity. It was found that after the first application of the positivity constraint the reconstructed image was already of a sufficiently high quality, and further conjugate-gradient iterations were not necessary.

Experiment. The references for our experiment were chosen to be significantly different to each other as well as those currently used in FTH. We begin by considering two cases, one with a partial circle and another with a 'cartoon-cloud' reference (Fig. 1a,b, respectively). The continuous curved features of these references differ markedly from the straight-edged features of corner holography and references constructed of pinholes. The continuous enclosure of an object by the reference structure deviates from the usual requirement of a reference that is isolated and contained to a small local region. This indicates how flexibly we can make use of the field-of-view.

For the general holographic method here, it is necessary that the spatial separation between some point on the reference and
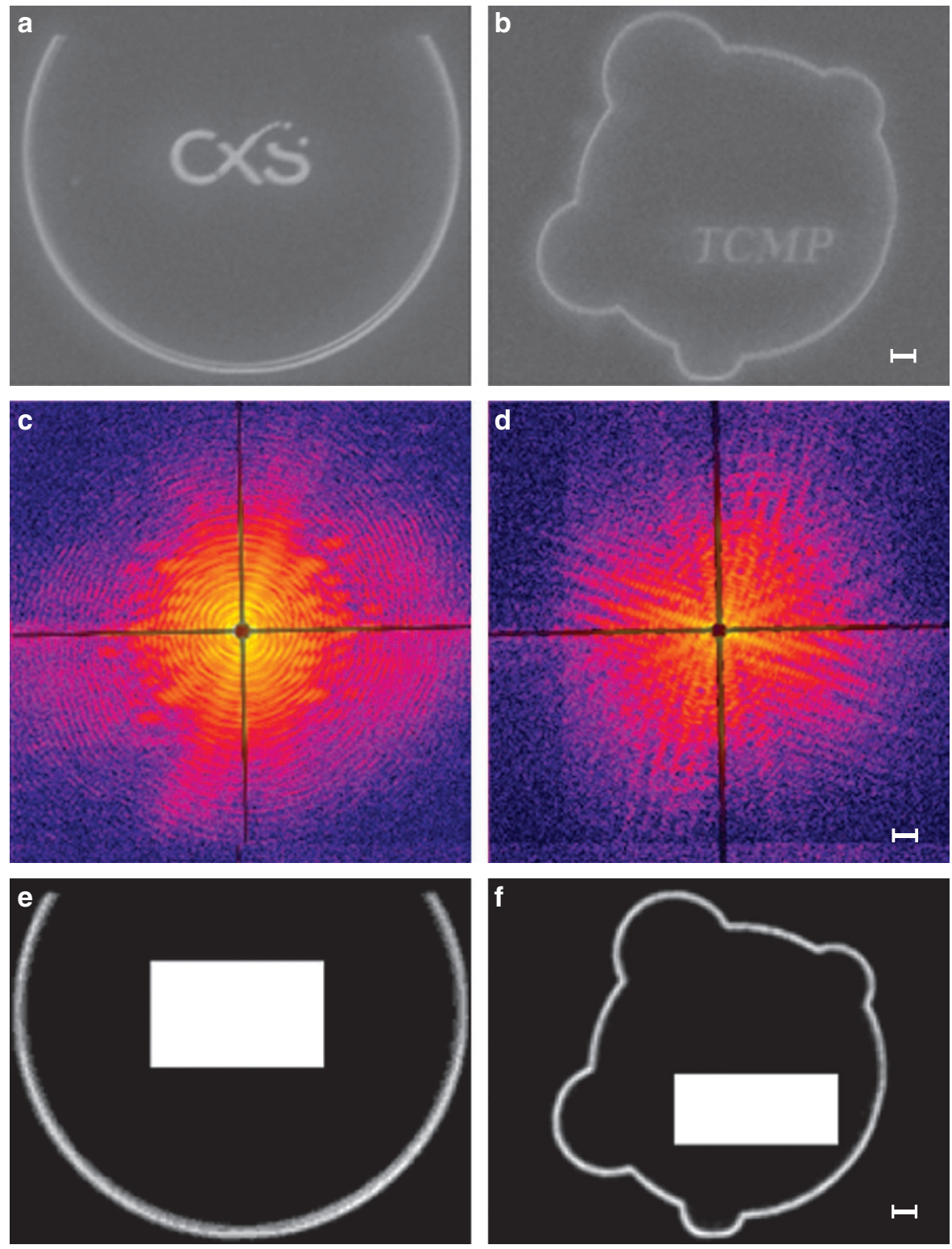

Figure 1 | Samples and diffraction patterns. The SEM images of the samples are shown in $\mathbf{a}, \mathbf{b}$. The diffraction patterns of the two samples are shown in $\mathbf{c}, \mathbf{d}$ with the central shaded areas indicating regions that have been set to zero for the reconstructions. The reference functions estimated from the SEM images are shown in e,f along with the assumed object areas indicated by the white rectangles. The scale bars in $\mathbf{b}, \mathbf{f}$ represent $500 \mathrm{~nm}$ and the scale bar in $\mathbf{d}$ represents $0.1 \mu \mathrm{m}^{-1}$. Images $\mathbf{a}, \mathbf{b}, \mathbf{e}$ and $\mathbf{f}$ are on the same scale. Images $\mathbf{c}$ and $\mathbf{d}$ are on the same scale. 
the object is at least of one object width in order to produce a sufficient number of linearly independent equations ${ }^{15}$. These requirements do not exclude references that approach or even overlap the sample, although knowledge of the reference in the overlap region will not contribute the coefficients of $\mathcal{M}$ in equation (3) and plays no part in the reconstruction procedure.

The experiment was performed at FERMI ${ }^{24-26}$, a seeded soft $\mathrm{X}$-ray free-electron laser. It is a highly suitable source for coherent $\mathrm{X}$-ray imaging because of its high brightness and excellent coherence properties ${ }^{8}$. Our test objects were fabricated on $\mathrm{Si}_{3} \mathrm{~N}_{4}$ windows using a focused ion beam (see Methods), similar to other CDI experiments ${ }^{8,27}$. Scanning-electron microscope (SEM) images of the two samples are shown in Fig. 1a,b. Diffraction patterns recorded from 50 and 100 exposures for the Coherent X-ray science (CXS) and Theoretical condensed matter physics (TCMP) samples are shown in Fig. 1c,d, respectively. Data contained large crossed streaks that arise from $\mathrm{X}$-ray scattering from the edges of the $\mathrm{Si}_{3} \mathrm{~N}_{4}$ window. These streaks needed to be masked before reconstruction and are indicated by the shaded central regions. While this removes a sizable fraction of data, it is comparable to the amount of missing data present when using modular detectors in XFEL experiments ${ }^{28}$. A missing mode analysis ${ }^{21}$ identified one weakly constrained mode for the 'CXS' object, and none for 'TCMP', indicating that the likelihood of reconstruction ambiguities due to the missing data are low (see Methods).

The test objects consisted of two distinct objects on each window, of which one was designated as the reference. An estimate of the reference for each case, shown in Fig. 1e,f, was made from the SEM image of the sample (see Methods). We assume that the average illumination over multiple exposures is uniform, ignoring the impact of the non-uniform illumination profile as discussed further in the Methods section. With ILRUFT the use of a tight support is not necessary. The size of the assumed object areas were overestimated and are shown in Fig. 1e,f by the white rectangles. The analysis of the exit-wave autocorrelation used to set up the ILRUFT reconstruction is discussed further below in Methods. It is crucial to have an accurate centring of the diffraction pattern and an accurate reference wave intensity to produce the highest-quality image. These parameters were refined by searching for values that minimized the residual error ${ }^{29}$. To further improve the robustness of the reconstruction, reality and positivity constraints were applied, as described above.

Applying the generalized holographic reconstruction procedure to the diffraction data produced the reconstructions for the test objects as shown in Fig. 2a,b. In both cases a clear image is recovered for each example test object.
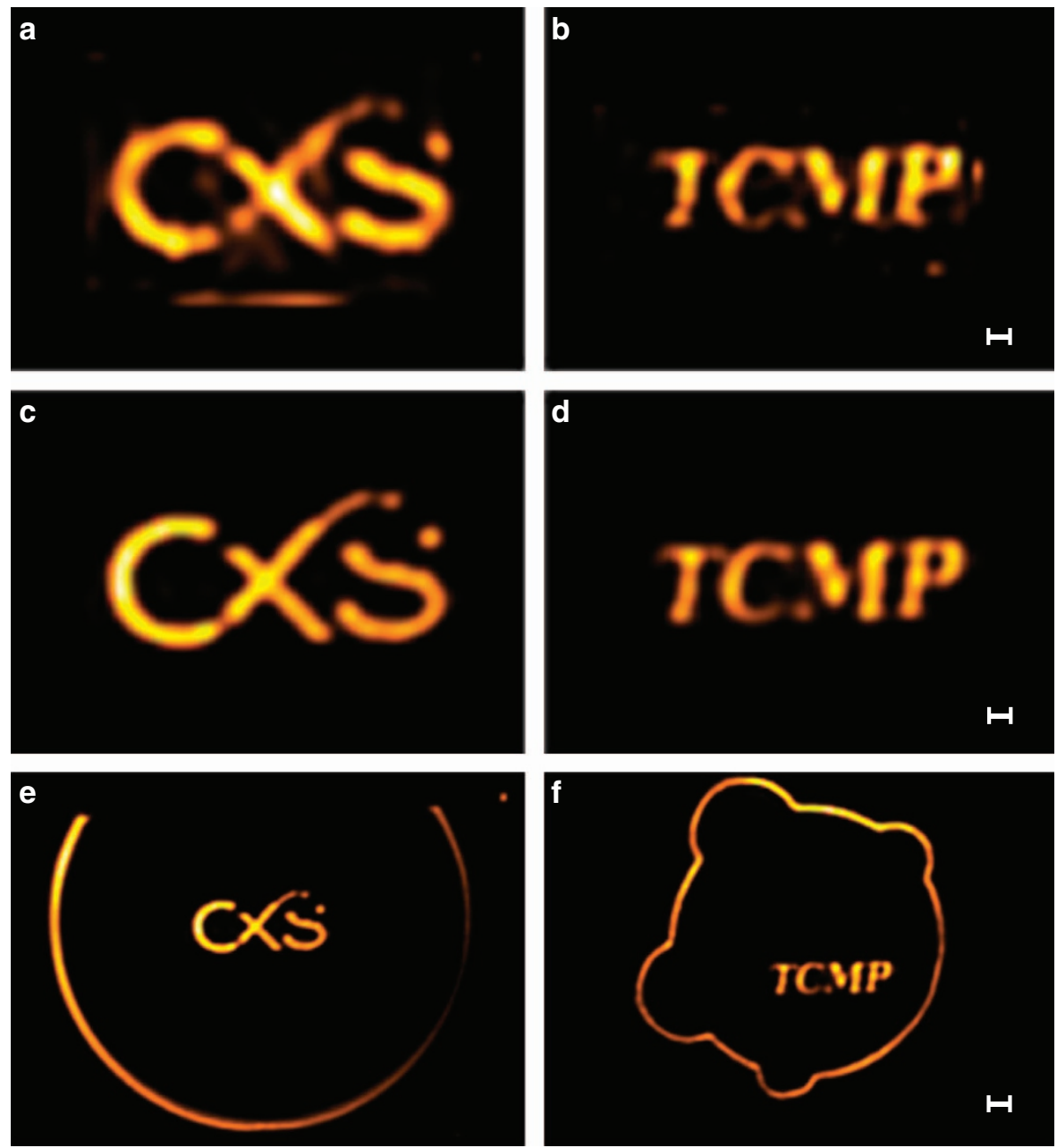

Figure 2 | Reconstructed images. The object intensities reconstructed by ILRUFT are shown in $\mathbf{a}, \mathbf{b}$ and those reconstructed by RAAR are shown in $\mathbf{c , d}$. The full exit surface wave intensity reconstructed by RAAR is shown in e,f. The scale bars shown in $\mathbf{b}, \mathbf{d}$ represent $200 \mathrm{~nm}$ and the scale bar in $\mathbf{f}$ represents $500 \mathrm{~nm}$. Images a-d are on the same scale. Images $\mathbf{e}$ and $\mathbf{f}$ are on the same scale. 
We compared the ILRUFT holographic reconstruction with a non-holographic nonlinear iterative CDI reconstruction. Successful reconstructions were obtained using the relaxed-alternatingaveraged reflection (RAAR) algorithm ${ }^{30}$, shown in Fig. $2 c, d$, which are very similar to the ILRUFT reconstructions. The estimated reference was provided as input, and the assumed object area was set to random values. The RAAR reconstruction used no a priori knowledge of the test object other than the reference wave. Seeding the RAAR without the SEM-derived reference wave resulted in failed reconstructions or slow convergence. The best reconstructions were obtained when RAAR was able to refine both reference and object and the initial support was allowed to vary using the shrinkwrap algorithm ${ }^{31}$. The refined exit surface waves produced by RAAR are shown in Fig. 2e,f.

Our general reconstruction method can be used with all of the references from existing FTH techniques. As an example we have performed a reconstruction with a uniformly redundant array (URA) reference, similar to that used by Marchesini et al. ${ }^{1}$ The measured diffraction pattern is shown in Fig. 3a and the SEM image of the sample is shown in Fig. 3b. There are in fact two URA structures, in addition to the 'CFEL' sample. As a single exposure was used to record the diffraction data, the intensity of the illumination was not uniform across the two URAs because of the beam profile, and the best results were obtained by selecting the brightest URA as the reference. The estimated reference and assumed object areas for the ILRUFT reconstruction are shown in Fig. 3c. The ILRUFT result, shown in Fig. 3d, is very similar to the RAAR reconstruction, shown in Fig. 3e. Owing to the missing data, reconstructing the object by convolution, as shown in ref. 1, is exceedingly challenging. To reduce the artifacts in the autocorrelation plane, a high-pass filter with a Guassian profile (see Methods) was applied to the diffraction pattern before performing the convolution. Our best result, shown in Fig. 3f, does not produce a clear image of either the 'CFEL' object or the URA object. Curiously, the centre of the convolved autocorrelation function, located in the top left corner of Fig. 3f, reproduces an image of the URA reference itself. This is the contribution from the $A_{\text {ref }}$ term in equation (2). The ILRUFT reconstruction demonstrates a distinct advantage for working with missing data over a reconstruction by convolution.

Each pixel in the images is $58 \mathrm{~nm}$ in width, and features across three pixels are clearly seen in all images, indicating a half-period resolution of $\sim 170 \mathrm{~nm}$. To confirm this estimate, the reconstruction was used to estimate the average diffraction intensity as a function of resolution shell and compared with the measured data in Fig. 4. The reconstructed diffraction intensity of both RAAR and ILRUFT reconstructions of the TCMP and CXS objects matches the measured intensity well to a half-period resolution of $170 \mathrm{~nm}$, at which point the reconstructed intensity drops, indicating higher-resolution information was not recovered. The resolution for the RAAR reconstruction was confirmed by constructing a phase retrieval transfer function ${ }^{32}$ from 80 trials with random initial objects. The phase retrieval transfer function (not shown) fell to a value of 0.5 at $150-\mathrm{nm}$ half-period resolution. The RAAR reconstruction intensity with the URA reference also extends to $\sim 170 \mathrm{~nm}$, but notably the ILRUFT reconstruction contains an intensity similar to the measured diffraction out to the edge of the detector.

The reference structures refined during the RAAR reconstructions display low-frequency variations of contrast that are inconsistent with the assumed uniform contrast used as input for the ILRUFT reconstructions. These features in the RAAR reconstruction may be because of the beam profile or because
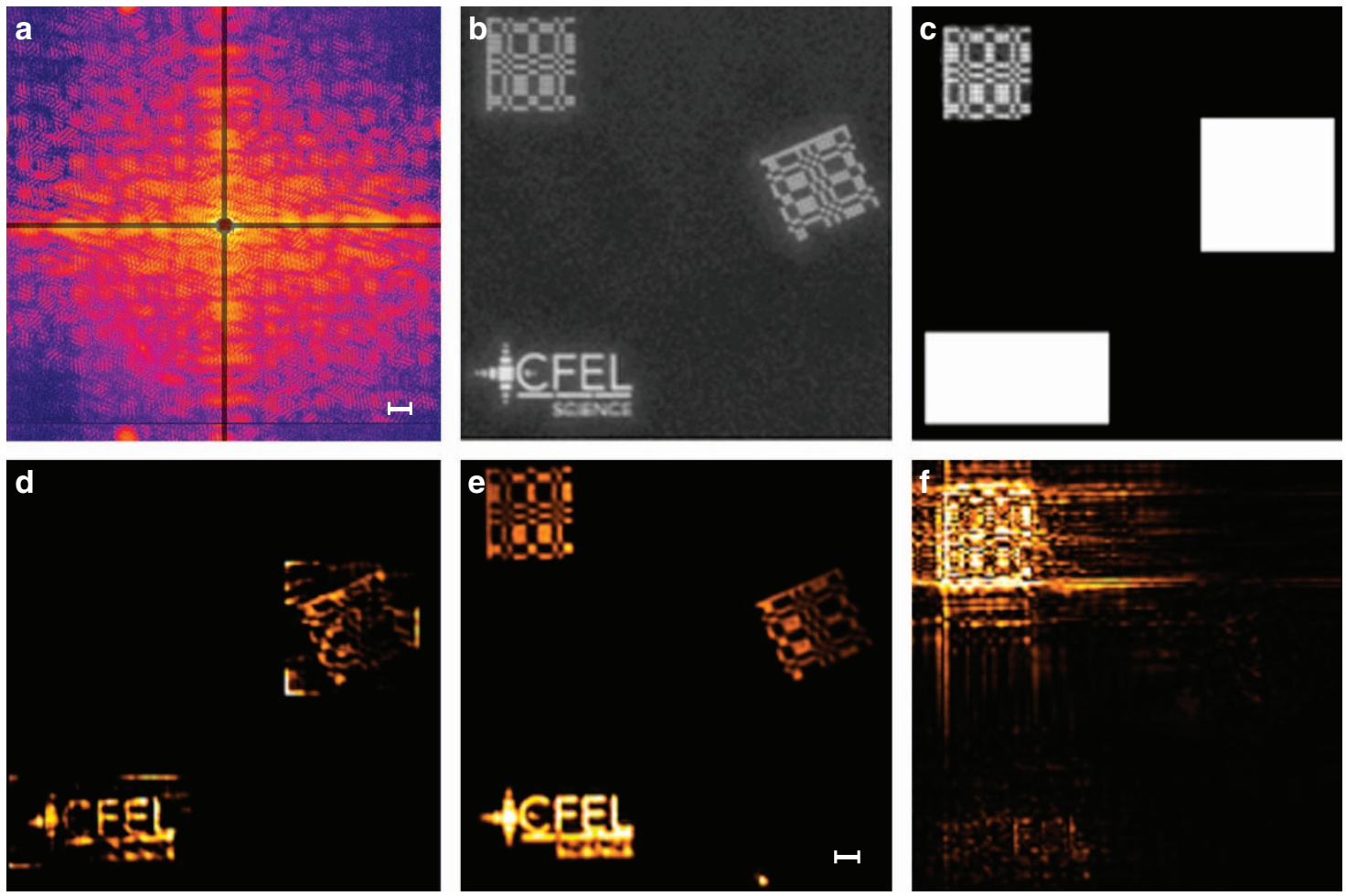

Figure 3 I URA reconstructions. The diffraction pattern of the URA reference case is shown in $\mathbf{a}$, and the SEM image of the sample is shown in $\mathbf{b}$. The estimated reference function is shown in $\mathbf{c}$, and the areas assumed to contain the objects are indicated by the white rectangles. The intensity of the ILRUFT reconstruction is shown in $\mathbf{d}$, the intensity of the averaged RAAR reconstruction is shown in $\mathbf{e}$ and the reconstruction by convolution is shown in $\mathbf{f}$. Note that $\mathbf{f}$ has been cropped so that the centre is located in the top left corner, so that only the most relevant part for comparison to the other reconstructions is shown. The scale bar in $\mathbf{a}$ represents $0.1 \mu \mathrm{m}^{-1}$. The scale bar in e represents $500 \mathrm{~nm}$ and images $\mathbf{b}-\mathbf{f}$ are on the same scale. 

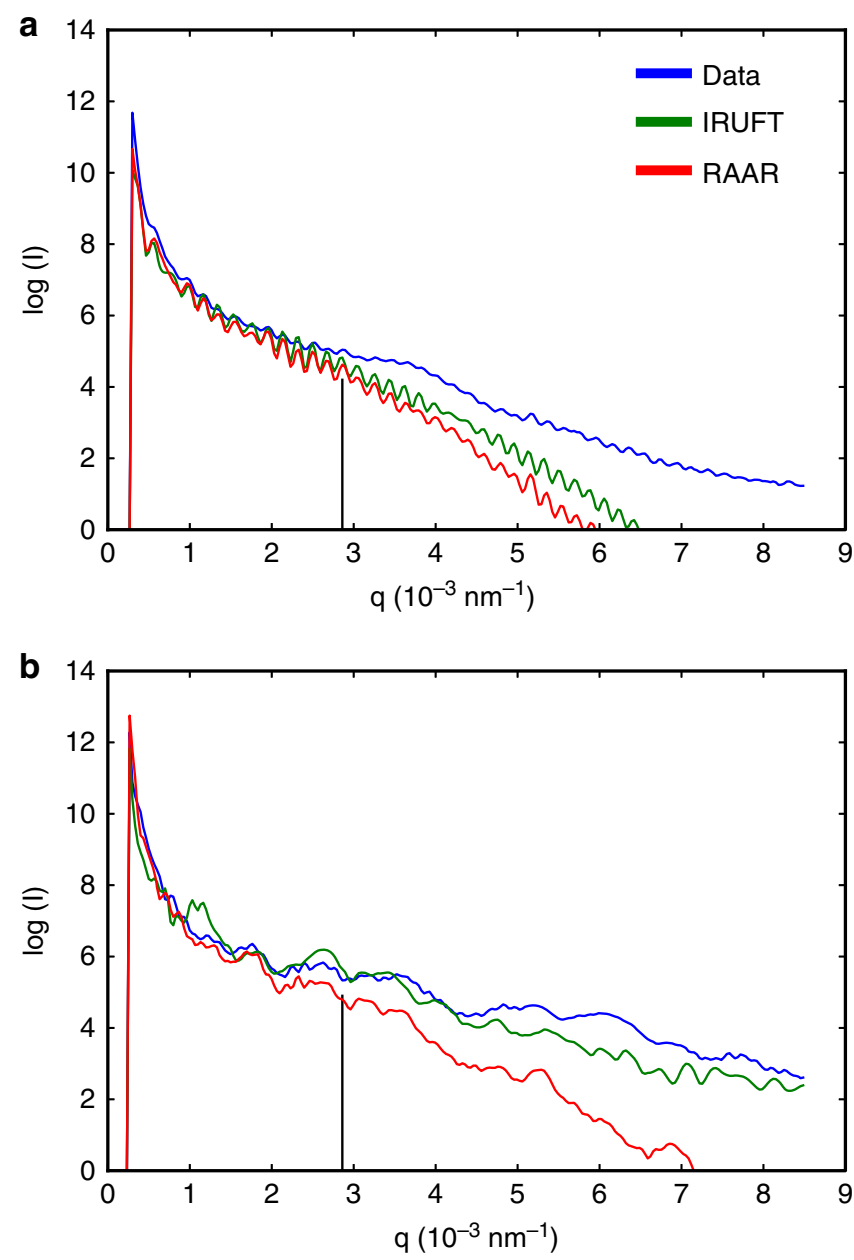

Figure 4 | Comparing measured and reconstructed diffraction intensities. The average intensity per detector pixel as a function of scattering vector $\mathrm{q}$ for the TCMP object (a) and the URA reference (b). The measured diffraction patterns are compared with the diffraction patterns modelled from the ILRUFT and RAAR reconstructions. The vertical black line indicates a full-period resolution of $350 \mathrm{~nm}$ ( $175 \mathrm{~nm}$ half-period resolution).

The plot for the CXS object is very similar to that for the TCMP object and has been omitted.

of missing data effects, which could be exacerbated by the large support required to cover both object and reference. Providing the refined reference from the RAAR reconstruction as an input for a new ILRUFT reconstruction did not produce any significant improvement.

There are one or two residual artifacts in the ILRUFT reconstructions, such as the horizontal line present below the 'CXS' (Fig. 2a), which indicate some remaining sources of error, such as measurement noise on the diffraction pattern, background scattering or inadequate reference characterization. Artifacts mostly occur at the edge or corners of the assumed object area as lines or dots not more than one or two pixels width. Hence, they are easily distinguished from the object's structure and do not complicate the interpretation of the image. This is supported by the fact that enlarging the object area shifts the location of these artifacts in reconstructions (not shown).

\section{Discussion}

ILRUFT differs from other holography methods by using a reference wave defined by a pixelated image rather than by assuming an ideal analytic form. The reference wave is sensitive to sample composition and thickness, in addition to the shape. Although this suggests that ILRUFT requires a higher level of $a$ priori knowledge than other holography methods, in practice a partial characterization of the reference wave does not cause ILRUFT to fail. The conjugate-gradient algorithm introduces elements of the solution with iteration in order of significance, so that the first iterations generally correspond to low-resolution reconstructions. An imprecise input reference wave introduces errors gradually with iteration number and still yields a resolution-limited image with a judicious termination of the algorithm. This is effectively the same regularization discussed previously to handle errors associated with measurement noise and missing data. In fact, if an accurate characterization of the reference wave is available, the ILRUFT method can be tailored to best use this information, potentially exceeding the performance of other methods. For example, the ILRUFT method could account for the aberrations of a Fresnel-zone plate reference ${ }^{13}$. It should be noted that other extended references, such as corner or URA references, impose requirements on the uniformity of reference composition and thickness. In this way, accurate reference fabrication is very important in holography generally. From the perspective of fabrication and a priori knowledge, a pinhole reference produces the simplest experiment because the hologram is readily interpreted as a blurred image of the object. However, with pinholes the trade-off between flux and resolution, as described above, is a significant disadvantage that motivates research into alternative holographic methods, where gains can be made by increasing experimental complexity.

Similar to other holographic methods, the resolution of the ILRUFT reconstruction is limited by the highest spatial frequency of the reference wave scattering. Consequently, an extended reference used with the ILRUFT method would be limited by the gradient of the edge profile, similar to references with sharp corners $^{12}$. The option is now available to design references that improve resolution in all directions simultaneously, avoiding the directional bias of corner references.

Aside from the structured reference scatterers, as shown here, the reference could be provided by a structured beam like the convergent beams that have been used in Fresnel CDI (ref. 33). If the propagation between the sample and the detector is a Fresnel propagator, then no fabricated reference is required for ILRUFT $^{34}$. In this case the form of the propagator provides the coefficients from which to construct $\mathcal{M}$.

\section{Methods}

Sample preparation. The reference and test objects were constructed using a focused ion beam to deposit platinum $(\sim 16 \%)$ and carbon $(\sim 84 \%)$ on a silicon nitride window. The $\mathrm{Si}_{3} \mathrm{~N}_{4}$ windows were 30 -nm thick and the deposited structures were of the order of 10 -nm thick.

Experimental methods. The pulses produced by FERMI had a wavelength of $32.5 \mathrm{~nm}$ and are estimated to be $100 \mathrm{fs}$ in duration. The focal spot of the beam was $\sim 15 \times 30 \mu \mathrm{m}^{2}$. After the gas attenuator the pulse energy was $10-15 \mu \mathrm{J}$. The beam was attenuated by a further $1.25 \%$ by aluminium filters. Final measurements were made by integrating multiple pulses to the same window. The number of integrated pulses was chosen to be less than saturation and differed for each sample. The 'CXS' and 'TCMP' measurements were accumulated from 50 and 100 pulses, respectively. The URA measurement was obtained with a single shot, with reduced beam attenuation so that the total post-filter energy was $10 \mu \mathrm{J}$. The diffraction patterns were acquired using a 32.5-nm multilayer mirror that reflects the scattered photons on a CCD detector (Princeton instrument MTE2048B, $13.5 \mu \mathrm{m}$ pixel size) placed at $\sim 55 \mathrm{~mm}$ from the interaction region. In order to avoid damage to the CCD camera from the direct FEL beam, the reflecting mirror has a hole of $\sim 0.5 \mathrm{~mm}$ diameter. Using such experimental configuration, the maximum scattering angle is of about $14 \mathrm{deg}$ with a missing data region of $0.4 \mathrm{deg}$. The maximum spatial frequency recorded by the detector was $2 \pi \times 0.017 \mathrm{~nm}^{-1}$.

Reconstruction methods. The reference was estimated from the SEM image by selecting the reference structure using a binary mask and applying a Gaussian noise 

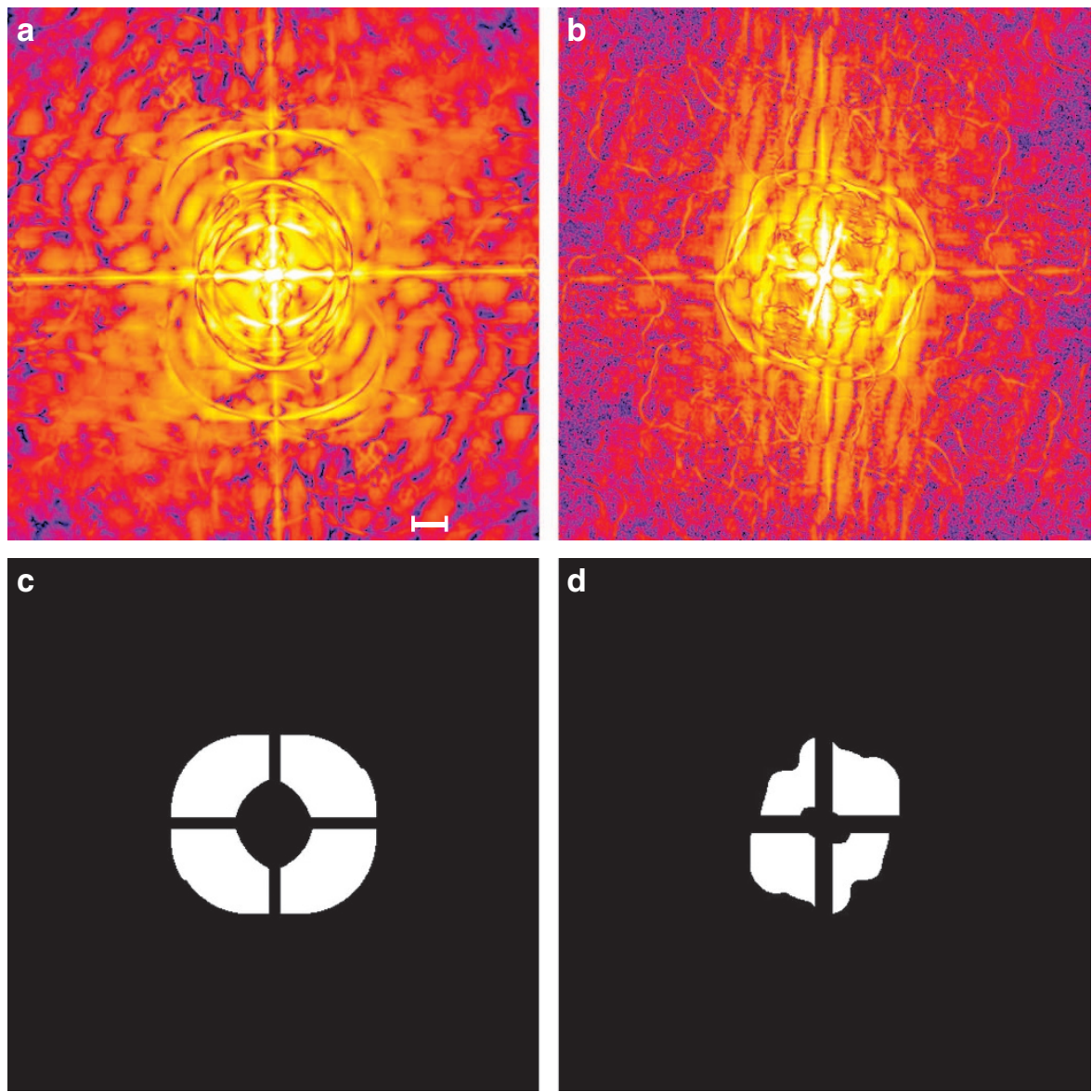

Figure 5 | Autocorrelation functions and linear equation regions. The amplitude of the autocorrelation functions generated from the inverse Fourier transform of the masked diffraction patterns (Fig. 1a,b) are shown in $\mathbf{a}, \mathbf{b}$ on a log scale, cropped to show only the relevant regions for holographic ILRUFT analysis. The regions of linear equations assumed for the analysis are shown in $\mathbf{c}, \mathbf{d}$. The scale bar in a represents $350 \mathrm{~nm}$ and all images are on the same scale.

filter. The filter was 2 pixels full-width at half-maximum. The reference size was scaled to match the size of features in the experimentally determined autocorrelation function (shown in Fig. 5a,b). The normalization of the reference was determined by minimizing the residual error of the ILRUFT reconstruction ${ }^{29}$.

The linear equation regions, shown in Fig. 5c,d, were estimated by taking a cross-correlation of the reference domain and the assumed object area. Horizontal and vertical streaks in the horizontal and vertical directions are due to the spread of the nonlinear term, $\mathcal{P} \otimes A_{\text {obj }}$, and were excluded from the linear equation region.

The ILRUFT reconstruction was run for 15 iterations and took the order of minutes to compute. It was terminated when the ratio of the change in object intensity to the change in residual error began to sharply increase. At this point, further updates to the reconstructed objects did not improve the comparison with the experimental data and represented the addition of noisy information to the images.

The RAAR reconstructions were run for 200 iterations, updating the support every 10 iterations. The RAAR feedback parameter $(\beta)$ was 0.9 . The shrinkwrap algorithm was implemented with a threshold of $15 \%$ of the maximum intensity of the object, after convolving with a Gaussian kernel. The half-width at halfmaximum of the Gaussian kernel was reduced linearly from three pixels to one pixel during the reconstruction. Reality and positivity constraints were not applied.

The convolution reconstruction with the URA reference was performed by convolving the experimentally determined exit-wave autocorrelation function with the estimated URA reference function (Fig. 3b). Before performing the convolution, a high-pass filter was applied to the autocorrelation function defined by $1-e^{-r^{2} /\left(2 \sigma^{2}\right)}$, where $r$ is a radial coordinate and $\sigma$ was set to an eighth of the detector width. The filter reduced artifacts in the exit-wave autocorrelation function because of the missing data region, and the optimal width of the filter was chosen by visual inspection of the result.

Data processing. The mean offset value of the detector and any uniform background scattering was estimated from a $100 \times 100$ pixel corner region of the diffraction pattern and subtracted. A mask was created to remove the streaks from the window scattering and the central hole. The diffraction pattern was centred by computing the centre of mass of the diffraction pattern and later refined by minimizing the residual error in the ILRUFT reconstruction.

Missing mode analysis. A missing mode analysis ${ }^{21}$ was performed using the object pixel basis to identify weakly constrained information about the object. Weakly constrained modes are functions that when added or subtracted from the reconstructed image have a minimal effect on the error metric minimized by the reconstruction algorithm. The reconstruction had one weakly constrained mode with a constraining power of 0.03 ; however, it had no apparent affect on the ILRUFT reconstruction after reality and positivity constraints were applied. The 'CXS' reconstruction with RAAR had no weakly constrained modes after the support was refined by shrinkwrap. The 'TCMP' reconstruction using ILRUFT had no weakly constrained modes and the minimum constraining power was 0.3 .

Integrating multiple exposures. When the diffraction is accumulated from multiple exposures (indexed by $i$ ) and the probe illumination fluctuates between exposures, the average cross-correlation between reference waves and object waves is

$$
\begin{aligned}
\left\langle C_{\mathrm{ref}, \mathrm{obj}}\right\rangle= & \left\langle\psi_{\mathrm{ref}, i}^{*} \otimes \psi_{\mathrm{obj}, i}\right\rangle+\left\langle\psi_{\mathrm{obj}, i}^{*} \otimes \psi_{\mathrm{ref}, i}\right\rangle \\
& \approx\left\langle\psi_{\mathrm{ref}, i}^{*}\right\rangle \otimes\left\langle\psi_{\mathrm{obj}, i}\right\rangle+\left\langle\psi_{\mathrm{obj}, i}^{*}\right\rangle \otimes\left\langle\psi_{\mathrm{ref}, i}\right\rangle .
\end{aligned}
$$

This case can be accommodated by defining $\psi_{\text {ref }}=\left\langle\psi_{\mathrm{re}, i}^{*}\right\rangle$ for the input to ILRUFT. The reconstructed object wave is then interpreted as the average of the object waves from each exposure. The second term affected by a variable probe is $A_{\text {ref, }}$ which is approximated by

$$
\left\langle A_{\mathrm{ref}, i}\right\rangle \approx\left\langle\psi_{\mathrm{ref}, i}^{*}\right\rangle \otimes\left\langle\psi_{\mathrm{ref}, i}\right\rangle .
$$

A source of pulse variability in our experiment was beam jitter in the sample plane and the average reference wave can correspond without the assumption of 
uniform reference contrast more closely than the reference wave for any individual exposure. Any errors that arise from these approximations are regularized by truncating the ILRUFT iteration as described above in the reconstruction methods.

\section{References}

1. Marchesini, S. et al. Massively parallel x-ray holography. Nat. Photonics 2, 560-563 (2008).

2. Gorniak, T. et al. X-ray holographic microscopy with zone plates applied to biological samples in the water window using 3rd harmonic radiation from the free-electron laser flash. Opt. Express 19, 11059-11070 (2011).

3. Eisebitt, S. et al. Lensless imaging of magnetic nanostructures by X-ray spectroholography. Nature 432, 885-888 (2004).

4. Gunther, C. M. et al. Microscopic reversal behavior of magnetically capped nanospheres. Phys. Rev. B 81, 064411 (2010).

5. Pfau, B. et al. Origin of magnetic switching field distribution in bit patterned media based on pre-patterned substrates. Appl. Phys. Lett. 99, 062502 (2011).

6. Wang, T. et al. Femtosecond single-shot imaging of nanoscale ferromagnetic order in $\mathrm{co}=$ pd multilayers using resonant $\mathrm{x}$-ray holography. Phys. Rev. Lett. 108, 267403 (2012).

7. Rosenhahn, A. et al. Digital in-line holography with femtosecond vuv radiation provided by the free-electron laser flash. Opt. Express 17, 8220-8228 (2009).

8. Capotondi, F. et al. Coherent imaging using seeded free-electron laser pulses with variable polarization: first results and research opportunities. Rev. Sci. Instrum. 84, 051301 (2013).

9. McNulty, I. et al. High-resolution imaging by Fourier-transform X-ray holography. Science 256, 1009-1012 (1992).

10. Schlotter, W. F. et al. Multiple reference fourier transform holography with soft x rays. Appl. Phys. Lett. 89, 163112 (2006).

11. Podorov, S. G., Pavlov, K. M. \& Paganin, D. M. A non-iterative reconstruction method for direct and unambiguous coherent diffractive imaging. Opt. Express 15, 9954-9962 (2007).

12. Guizar-Sicairos, M. \& Fienup, J. R. Holography with extended reference by autocorrelation linear differential operation. Opt. Express 15, 17592-17612 (2007).

13. Geilhufe, J. et al. Monolithic focused reference beam x-ray holography. Nat. Commun. 5, 3008 (2014).

14. Zhu, D. et al. High-resolution $\mathrm{x}$-ray lensless imaging by differential holographic encoding. Phys. Rev. Lett. 105, 043901 (2010).

15. Martin, A. V. \& Allen, L. J. Direct retrieval of a complex wave from its diffraction pattern. Opt. Commun 281, 5114-5121 (2008).

16. D’Alfonso, A. J., Morgan, A. J., Martin, A. V., Quiney, H. M. \& Allen, L. J. Fast deterministic approach to exit-wave reconstruction. Phys. Rev. A. 85, 013816 (2012).

17. Hestenes, M. R. \& Stiefel, E. Methods of conjugate gradients for solving linear systems. J. Res. Nat. Bur. Stand 49, 409 (1952).

18. Morgan, A. J. et al. Fast deterministic single-exposure coherent diffractive imaging at subangstrom resolution. Phys. Rev. B 87, 094115 (2013).

19. D’Alfonso, A. J. et al. Deterministic electron ptychography at atomic resolution. Phys. Rev. B 89, 064101 (2014).

20. Miao, J. et al. Quantitative image reconstruction of gan quantum dots from oversampled diffraction intensities alone. Phys. Rev. Lett. 95, 085503 (2005).

21. Thibault, P., Elser, V., Jacobsen, C., Shapiro, D. \& Sayre, D. Reconstruction of a yeast cell from x-ray diffraction data. Acta Crystallogr. A A62, 248-261 (2006).

22. Lawson, C. L. \& Hanson, R. J. Solving Least Squares Problems (Prentice-Hall, 1987).
23. Bro, R. \& Jong, S. D. A fast non-negativity-constrained least squares algorithm. J. Chemometr. 11, 393-401 (1997).

24. Allaria, E. et al. The fermi@elettra free-electron-laser source for coherent x-ray physics: photon properties, beam transport system and applications. New J. Phys. 12, 075002 (2010).

25. Allaria, E. et al. Highly coherent and stable pulses from the FERMI seeded free-electron laser in the extreme ultraviolet. Nat. Photonics 6, 699-704 (2012).

26. Allaria, E. et al. Two-stage seeded soft-x-ray free-electron laser. Nat. Photon 7 , 913-918 (2013).

27. Chapman, H. N. et al. Femtosecond diffractive imaging with a soft-X-ray freeelectron laser. Nat. Phys. 2, 839-843 (2006).

28. Seibert, M. M. et al. Single mimivirus particles intercepted and imaged with an $\mathrm{x}$-ray laser. Nature 470, 78-81 (2011).

29. Morgan, A. J. et al. High-fidelity direct coherent diffractive imaging of condensed matter. Phys. Rev. B 84, 144122 (2011).

30. Luke, D. R. Relaxed averaged alternating reflections for diffraction imaging Inverse Probl. 21, 37-50 (2005).

31. Marchesini, S. et al. X-ray image reconstruction from a diffraction pattern alone. Phys. Rev. B 68, 140101 (2003).

32. Chapman, H. N. et al. High-resolution ab initio three-dimensional x-ray diffraction microscopy. J. Opt. Soc. Am. A 23, 1179-1200 (2006).

33. Williams, G. et al. Fresnel coherent diffractive imaging. Phys. Rev. Lett. 97, 025506 (2006).

34. Morgan, A. J., Martin, A. V., D’Alfonso, A. J., Putkunz, C. T. \& Allen, L. J. Direct exit-wave reconstruction from a single defocused image. Ultramicroscopy 111, 1455-1460 (2011)

\section{Acknowledgements}

We thank Professor L.J. Allen for useful discussion and feedback. This research was supported under the Australian Research Council's Centre of Excellence programme and DECRA funding schemes (Project Nos DE130100739 and DE140100624). FERMI project of Elettra Sincrotrone Trieste is partially supported by the Italian Ministry of University and Research under grant numbers FIRB-RBAP045JF2 and FIRB-RBAP06AWK3 and by the grant from Friuli Venezia Giulia Region: Nanotox 0060-2009. F.C., E.P. and L.R. thank N. Mahne, C. Svetina, M. Zangrando and the Fermi Commissioning Team for the valuable technical support during the measurement preparation.

\section{Author contributions}

The reconstruction theory and methods were developed by A.V.M. and A.J.D. The samples were designed by A.V.M. and F.W. and they were prepared by F.S. The experiment was performed by F.W., R.B., F.C., R.A.K., E.P., L.R. and C.H.Y. in consultation with H.N.C. The reconstructions were performed by A.V.M. and A.J.D who also wrote the manuscript with input from all authors.

\section{Additional information}

Competing financial interests: The authors declare no competing financial interests.

Reprints and permission information is available online at http://npg.nature.com/ reprintsandpermissions/

How to cite this article: Martin, A. V. et al. X-ray holography with a customizable reference. Nat. Commun. 5:4661 doi: 10.1038/ncomms5661 (2014). 


\section{Corrigendum: X-ray holography with a customizable reference}

Andrew V. Martin, Adrian J. D'Alfonso, Fenglin Wang, Richard Bean, Flavio Capotondi, Richard A. Kirian, Emanuele Pedersoli, Lorenzo Raimondi, Francesco Stellato, Chun Hong Yoon \& Henry N. Chapman

Nature Communications 5:4661 doi: 10.1038/ncomms5661 (2014); Published 22 Aug 2014; Updated 16 Oct 2014

The original version of this Article contained an error in the spelling of the author Emanuele Pedersoli, which was incorrectly given as Emmanuele Pedersoli. This has now been corrected in both the PDF and HTML versions of the Article. 